\title{
HIGH ENERGY PHENOMENA IN AGN JETS
}

\author{
F. TAKAHARA \\ Department of Earth and Space Science, Osaka University \\ Toyonaka, Osaka 560, Japan
}

\section{Introduction}

BL-Lac objects and optically violent variable quasars (OVVs), called together blazars, are characterized by rapid time variability, strong optical polarization, superluminal expansion and strong gamma-ray emission. Such properties are understood in the framework of a relativistic jet emanated from the central powerhouse. Blazars are considered to be objects for which the direction of the jet is very close to the line of sight.

Four major issues are addressed on the physics of blazars. The first one is the bulk acceleration mechanism of the jets; radiative acceleration has been shown to be ineffective as a result of strong radiation drag and magnetic acceleration is still ad hoc and not a compelling one. The second one is the energetics of the jets; do particles or magnetic fields dominate the dynamics of jets? The third issue is the composition of the jets; is the jet composed of ususal proton-electron plasma or electron-positron plasma? The fourth issue is the particle acceleration mechanism in the jets; we need an efficient and ubiquitous mechanism to explain observations of blazars.

Important keys to these questions are provided by recent gamma-ray and multiwavelength observations. As is well known, to explain rapid time variabilities and gamma-ray transparency, we need relativistic beaming and a small size of the gamma-ray emitting region [1]. The inferred size of the gamma-ray emitting region is around $0.003 \mathrm{pc}$ to $0.03 \mathrm{pc}$. This implies that gamma-ray emission and bulk acceleration of jets take place at a few hundred Schwardschild radii unless the ratio of the size to the distance from the central black hole is extremely small. Thus, the gamma-ray emission probes deep inner part of the relativistic jets, one or two orders of magnitudes deeper than the radio VLBI observations. 
Broad band emission spectra from blazars are composed of distinctly two components. While radio through optical emission is believed to be produced by the synchrotron radiation of relativistic electrons, gamma-rays are most naturally produced by the inverse Compton scattering of soft photons by the same electron population $[1,2,3,4]$. X-ray emission is due to either synchrotron radiation or inverse Compton depending on the sources. In the following, I discuss implications for the electron acceleration mechanism and the nature of the relativistic jets implied by these observations. Here, I only describe the main results; the details can be seen in $[5,6,7]$.

\section{ELECTRON ACCELERATION}

As for the particle acceleration mechanism, diffusive shock acceleration has shown some success in reproducing the observed radiative properties of blazars, although its simple application to electron acceleration in AGN environments may not be so trivial. Comparing acceleration time scale, radiative cooling time and advection time, we expect the universal power law spectrum with an index of 2 below the break Lorentz factor $\gamma_{\mathbf{b r}}$ and a power law spectrum with an index of 3 above $\gamma_{\mathrm{br}}$. The resultant emission spectra of synchrotron radiation and inverse Compton scattering of external soft photons are a power law with an energy index of 1.0 at high energies, while 0.5 at lower energies. Those break features may be indentified with the $\mathrm{MeV}$ break observed for many of OVV quasars. Note that since the synchrotron self-Compton spectra are rather smooth, reflecting the broad energy distribution of target photons, a clear break will be seen only for Compton scattering of external photons.

The derived spectral shape implies that the dissipated energy into the particle acceleration is efficienty converted into the beamed radiation, because for the universal energy spectra the dissipated energy is equally shared among electrons with a unit logarithmic energy scale.

Note that while in most of other works, electron spectra are arbitrarily assumed to fit the observed spectra, the standpoint here is that the shock acceleration can well reproduce such spectra provided that the shock velocity is near the speed of light.

\section{MODEL PREDICTIONS}

For simplicity, adopting a uniform sphere of a radius $R$ with a bulk Lorentz factor of $\Gamma$ and assuming given energy densities of magnetic field $u_{\text {mag }}$, relativistic electrons $u_{\text {rel }}$ and external soft photons $u_{\text {ext }}$, we can predict the energy densities of various radiation components [6,7]. It is found that as long as the shock is relativisitic, energy density of the radiation becomes comparable to that of electrons, which means a large radiative efficiency. 
An important inference can be obtained for the classification of radiative properties of relativisic jets based on the relative importance of $u_{\text {rel }}$ and $u_{\text {mag. }}$. When there are negligible external photons, we have an SSC dominated source if $u_{\text {rel }}$ dominates over $u_{\text {mag }}$, while we have a synchrotron dominated source if $u_{\text {mag }}$ dominates over $u_{\text {rel }}$. Effect of external soft photons is also easily incorporated. When $u_{\text {ext }}$ dominates over $u_{\text {mag }}$, external Compton always dominates over synchrotron radiation. The relative importance SSC and external Compton luminosities is determined by the ratios of $u_{\text {rel }}$ to $u_{\text {mag }}$ and $u_{\text {mag }}$ to $u_{\text {ext }}$. Generally we have Compton dominated sources for a large ratio of $u_{\text {rel }}$ to $u_{\text {mag }}$. Thus the radiative properties are neatly classified according to the relative importance of energy densities of magnetic field, particles and external photons.

Some limiting cases deserve further examinations. For sources in which cooling is dominated by synchrotron radiation, the jet is dominated by the Poynting flux and is not radiatively decelerated. For sources cooling mainly through SSC, the dominant power is particle kinetic flux but the jet does not suffer from strong radiation drag, either. When external Compton radiation dominates, jets suffer from strong radiation drag and are decelerated.

Above arguments are further extended to the estimation of the jet power. The power of each component is determined by $L_{i}=\pi R^{2} c u_{i} \Gamma^{2}$ except for the external soft photons. For the radiation components, $L_{i}$ is smaller than the observed isotropic luminosity $L_{i, \mathrm{obs}}$ by a factor of $4 \delta^{2}$ if we take $\delta \approx \Gamma$, where $\delta$ is the beaming factor. Because of efficient radiative cooling, as far as the shock is relativistic, the kinetic power is equal to the radiative power within a factor of a few.

\section{OBSERVATIONAL COMPARISONS}

Conversely, we can infer the properties of the gamma-ray emission region from multiwavelength data. Applying to typical objects such as 3C279 and Mkn421, we have some general results. First, the observed properties are consistent with predictions of diffusive particle acceleration by (mildly) relativistic shocks. Second, kinetic power is about 30 times greater than the Poynting power, so the jet is kinetic power dominated. When we compute the kinetic power, we only take account of relativistic electrons with $\gamma_{\min }=1$. This puts a strong constraint on the magnetic mechanism of the bulk acceleration of jets. Third, the derived kinetic power amounts to about $10 \%$ of the Eddington luminosity for a typical black hole mass. If the jet is composed of electrons and protons, associated proton power would succeed the Eddington luminosity by a few orders of magnitudes, which may suggest that the jet is composed of electron positron pairs. One caveat is the value of $\gamma_{\min }$; if $\gamma_{\min }$ is as large as 100 , the proton power would be comparable to 
the Eddington luminosity, which may be reasonable. Detailed observations of the region, where electrons with Lorentz factor of 1 to 100 are resposible, will discriminate these two cases. Also the kinetic power derived from radio observations should be compared.

Since the model predicts that $\gamma_{\max }$ and $\gamma_{\mathrm{br}}$ are inversely proportional to the energy density of soft photons, $\mathrm{TeV}$ emission is expected for low luminosity objects with a high cutoff frequency of the synchrotron radiation. These sources roughly correspond to X-ray selected BL Lac objects. OVV quasars reveal a high luminosity with a low cutoff frequency of synchrotron radiation.

\section{CONCLUDING REMARKS}

I discussed several theoretical aspects of relativistic jets in AGNs, motivated by gamma-ray observations of blazars. Physical conditions of the jets are deduced by analyzing multiwavelength spectra in terms of the synchrotron and inverse Compton model. The results show that the jets are particle dominated and that they are largely comprised of electron-positron pairs. The electron spectra are explained by diffusive shock acceleration with radiative cooling. Maximum energy of electrons turns out to be as high as $\mathrm{TeV}$ only for low luminosity objects, while it is an order of $10 \mathrm{GeV}$ for high luminosity objects. I show that radiative properties of the jets are neatly classified by the relative importance between the kinetic and Poynting power.

\section{Acknowledgements}

This work is supported in part by the Scientific Research Fund of the Ministry of Education, Science and Culture under Grant Nos. 09640323 and 09223215.

\section{References}

1. Maraschi L., Ghisellini G. and Celotti A. 1992, Ap.J.L., 397, L5.

2. Sikora M., Begelman M. C. and Rees M. J. 1993, Ap.J., 421, 153.

3. Dermer D. C. and Schlickeiser R. 1993, Ap.J., 416, 458.

4. Inoue S. and Takahara F. 1996, Ap.J., 463, 555.

5. Takahara F. 1994, in Towards a Major Atmospheric Cerenkov Detector III, ed. T. Kifune (Tokyo: Universal academy Press) 131.

6. Takahara F. 1996, J.Korean A.S., 29, S99.

7. Takahara F. 1997, to appear in "Relativistic Jets in AGNs", (Lecture Notes in Physics, Springer). 